\title{
Efeitos a curto prazo da plataforma vibratória e oscilatória em indivíduos saudáveis
}

\author{
Short-term effects of whole body vibration and body oscillation in healthy subjects
}

\author{
Ulysses da Silva a, Paula Caitano Fontela ${ }^{b}$, Elenita Costa Beber Bonamigo ${ }^{c}$, Eliane Roseli Winkelmann ${ }^{d}$ \\ a Fisioterapeuta graduado pela Universidade Regional do Noroeste do Estado do Rio Grande do Sul (UNIJUí), ljuí, RS, Brasil. \\ b Fisioterapeuta. Mestranda do Programa de Pós-Graduação em Ciências Pneumológicas da Universidade Federal do Rio Grande do Sul, Porto Alegre, RS, Brasil. \\ Fisioterapeuta. Mestre em Ciências do Movimento Humano pela Universidade do Estado de Santa Catarina. Docente do Departamento de Ciências da Vida da UNIJUí, \\ ljuí, RS, Brasil. \\ d Fisioterapeuta. Doutora em Ciências da Saúde pela Universidade Federal do Rio Grande do Sul. Docente do Programa de Pós-Graduação Latu Sensu Mestrado \\ em Atenção Integral à Saúde - PPGAIS e do Departamento de Ciências da Vida da UNIJUÍ, Ijuí, RS, Brasil.
}

RESUMO

Introdução: As plataformas vibratória e oscilatória vêm sendo utilizadas como recurso terapêutico em diferentes populações. No entanto, seus efeitos hemodinâmicos, respiratórios e ventilatórios são pouco conhecidos.

Objetivo: Avaliar os efeitos hemodinâmicos, respiratórios e ventilatórios durante o exercício na plataforma nas modalidades vibratória e oscilatória em indivíduos saudáveis e comparar os efeitos entre as modalidades terapêuticas.

Materiais e Métodos: Foi realizado um ensaio clínico cruzado. A amostra foi constituída por dez indivíduos saudáveis, cinco homens e cinco mulheres, com idade entre 18 a 30 anos. Os indivíduos foram submetidos a um protocolo de exercício na plataforma vibratória e após na oscilatória, (com período de intervalo entre as modalidades), durante um período de nove minutos, sendo usadas três posições ortostáticas variando a flexão do joelho entre $120^{\circ}, 150^{\circ}$ e $180^{\circ}$, permanecendo um minuto em cada posição, repetindo-se três vezes a sequência de exercícios. Variáveis hemodinâmicas, respiratórias e ventilatórias foram coletadas no início, durante e após três minutos de recuperação do exercício.

Resultados: Verificou-se alterações fisiológicas durante o exercício nas variáveis hemodinâmicas (PAS e FC) e ventilatórias $\left(\mathrm{VO}_{2}\right.$ e $\left.\mathrm{VCO}_{2}\right)$ nas plataformas vibratória e oscilatória. Ao comparar o exercício entre as modalidades da plataforma, observou-se que a oscilatória tende a provocar um maior aumento, comparada a vibratória, no $\mathrm{VO}_{2}$, $\mathrm{VCO}_{2}$ e potência circulatória.

Conclusão: O exercício realizado nas duas modalidades de plataforma não apresentou alterações significativas, além das esperadas durante a realização de um exercício, nas respostas hemodinâmicas, respiratórias e ventilatórias em indivíduos jovens e saudáveis.

Palavras chaves: equipamento; terapia por exercício; vibração, hemodinâmica.

\begin{tabular}{l|l} 
ABSTRACT & Introduction: Whole body vibration and body oscillation have been used as a therapeutic resource in different populations. However,
\end{tabular} hemodynamic, respiratory and ventilatory effects are less known.

Objective: To evaluate the hemodynamic, respiratory and ventilatory effects during exercise on the platform in both vibratory and oscillatory modes in healthy individuals and to compare the effects between the two therapeutic modalities.

Materials and Methods: A crossover clinical trial was performed. Ten healthy individuals were included, five men and five women, aged from 18 to 30 years. The subjects underwent an exercise protocol on the vibrating platform and after on the oscillatory mode (with an interval between modes) over a period of nine minutes. Three upright positions with knee flexion between 120,150 and 180 degrees were used, remaining one minute in every position and repeating three times the exercise sequence. Hemodynamic, respiratory and ventilatory variables were collected at the beginning, during, and after three minutes of recovery from exercise.

Results: Physiological changes during exercise were found in hemodynamic (SBP and HR) and respiratory variables $\left(\mathrm{VO}_{2}\right.$ and $\left.\mathrm{VCO}_{2}\right)$ in the vibrating and oscillating platform. When comparing the performance between the platform modes, it was observed that the oscillatory mode tends to cause a greater increase, compared to vibration, in the $\mathrm{VO}_{2}, \mathrm{VCO}_{2}$ and circulatory power.

Conclusion: The exercise performed in the two platform modes had no significant changes in hemodynamic, respiratory and ventilatory responses other than those expected during an exercise in young healthy individuals.

Keywords: equipment; exercise therapy; body vibration, hemodynamics.

\section{Correspondência:}

ELIANE ROSELI WINKELMANN

Rua do Comércio, 3000 - Bairro Universitário

Departamento de Ciências da Vida - DCVida - UNIJUÍ

98700-000 ljuí, RS, Brasil

E-mail: elianew@unijui.edu.br 


\section{INTRODUÇÃO}

A utilização da vibração surgiu por volta da década de 1970, na antiga União Soviética e tinha como objetivos combater a perda da massa óssea e a diminuição muscular, causadas pela hipogravidade, durante a permanência dos astronautas no espaço, efeito este obtido pela manutenção da fisiologia normal do sistema osteomuscular gerado pela aceleração a que o corpo é submetido durante o movimento de subida e descida da vibração ${ }^{1}$. O uso diário por dez minutos da máquina de vibração nos membros proporcionava aos astronautas soviéticos a chance de permanência no espaço por 3,5 vezes mais tempo, chegando a cerca de 420 dias, contra no máximo 120 dias dos astronautas americanos ${ }^{1}$. A vibração foi utilizada inicialmente como sistemas de cabos que eram fixados em peças sobre a pele, transmitindo a vibração de forma local. Com o avanço da tecnologia, foi se construindo aparelhos para trabalhar os músculos de uma forma global, conseguindo trazer o efeito de vibração por todo o corpo, dispensando um menor tempo e energia elétrica, surgindo assim a plataforma vibratória, chamado de Whole-body vibration (WBV) 1 .

A necessidade da adoção de hábitos saudáveis tem sido discutida como forma de combater os danos causados à saúde. O treinamento aeróbio é uma ferramenta não medicamentosa de maior acessibilidade e eficiência para promoção e manutenção da saúde ${ }^{2}$. O exercício físico regular proporciona inúmeros benefícios para a saúde com tais benefícios tipicamente variando de acordo com o volume, assim como, pela intensidade, duração e frequência. A evidência sugere uma relação dose-resposta tais que estar ativo, mesmo a um nível modesto, é preferível a estar inativo ou sedentário. Maiores benefícios são comumente associados com o indivíduo previamente sedentário assumindo um estilo de vida mais ativo. Existe uma relação linear aparente entre exercício físico e estado de saúde e, como regra geral, o aumento em exercício físico e aptidão resulta em melhorias adicionais no estado de saúde ${ }^{3}$.

No Brasil, apenas $13 \%$ da população realiza o mínimo recomendado de 30 minutos (min.) diários de exercício físico contínuo em um ou mais dias da semana, e somente $3,3 \%$ desses realizam exercício superior a $30 \mathrm{~min}$. em cinco ou mais dias da semana ${ }^{4}$. Para que o exercício físico seja realizado com segurança é importante que seus efeitos sejam conhecidos. Alguns equipamentos para a realização de exercícios físicos têm seus efeitos amplamente divulgados, como a esteira ergométrica e o cicloergômetro. Atualmente um novo equipamento vem sendo utilizado, a plataforma vibratória e oscilatória, cujos efeitos não são bem conhecidos. Os estudos têm abordado os efeitos da vibração no sistema musculoesquelético ${ }^{5,6,7}$, porém sabe-se pouco sobre a influência deste estímulo mecânico nas variáveis hemodinâmicas, respiratórias e ventilatórias.

Nos últimos anos a vibração tem sido utilizada como uma forma de treinamento para idosos, população sujeita a riscos de quedas. Embora alguns estudos mostram benefícios da plataforma vibratória ${ }^{6}$, por meio da melhora da força, densidade mineral óssea e do equilíbrio em idosos, outras questões não são abordadas, tais como parâmetros ventilatórios e hemodinâmicos. Pelo fato de ser um treinamento global, é aceitável supor que ele interfira não somente no sistema osteomuscular, mas também em outros sistemas, como o cardiovascular e respiratório.

A WBV tem sido descrita como uma forma de treinamento que produz respostas semelhantes ao exercício de resistência sobre a força muscular ${ }^{8}$, com respostas fisiológicas que estabelecem relação com diferentes níveis do organismo, proporcionando estímulos aos receptores cutâneos e fusos musculares, tendo respostas de reflexo tônico vibratório podendo ser utilizada em qualquer faixa etária.

Outra modalidade de exercício é a plataforma oscilatória, baseada no treinamento proprioceptivo em superfícies estáveis e instáveis, que vem sendo rotineiramente usado em treinamento físico, tendo como principal objetivo melhorar a acuidade proprioceptiva e a estabilidade articular dinâmica ${ }^{9,10,11}$. Para a realização deste treinamento é utilizado como base de sustentação do corpo, o solo ${ }^{12}$, sendo uma superfície estável, mas também pode se utilizar equipamentos específicos, como os que proporcionam bases instáveis ${ }^{13}$. Estes equipamentos consistem de plataformas que se caracterizam por proporcionar diferentes condições de instabilidade. Cada tipo de equipamento pode proporcionar um estímulo perturbatório diferente ao corpo humano, obtendo diferenças quanto a geometria da plataforma, material de fabricação, suas restrições mecânicas, posição adotada no teste, posição ortostática tanto bipodal quanto unipoda $\mid{ }^{14}$ podendo influenciar na relevância da instabilidade que é induzida pelo exercício.

Conhecer os efeitos destas modalidades de treinamento físico, pode fornecer os parâmetros de segurança necessários para que os mesmos sejam opções de exercício físico para diferentes populações. Portanto, o objetivo deste estudo foi avaliar os efeitos hemodinâmicos, respiratórios e ventilatórios durante o exercício na plataforma nas modalidades vibratória e oscilatória em indivíduos saudáveis e comparar os efeitos entre as modalidades terapêuticas.

\section{MATERIAIS E MÉTODOS}

Ensaio clínico cruzado realizado com uma amostra constituída por conveniência de dez indivíduos saudáveis. 
O estudo foi aprovado pelo Comitê de Ética em Pesquisa da Universidade Regional do Noroeste do Estado do Rio Grande do Sul - UNIJUí (n 814.925/2014).

A amostra foi composta por cinco homens e cinco mulheres, com idade entre 18 e 30 anos, estudantes da Universidade Regional do Noroeste do Estado do Rio Grande do Sul-UNIJUí, sem histórico de doenças cardíacas, respiratórias e ortopédicas e que concordaram em participar do estudo assinando o termo de consentimento livre e esclarecido.

O protocolo de pesquisa constou na avaliação hemodinâmica [pressão arterial sistólica (PAS) e diastólica (PAD) e frequência cardíaca $(\mathrm{FC})$ ]; respiratória [saturação periférica de oxigênio $\left(\mathrm{SpO}_{2}\right)$ e avaliação do esforço percebido (escala de Borg de membro inferior e da dispnéia)]; e ventilatória [ventilação minuto $(\mathrm{VE})$, consumo de oxigênio $\left(\mathrm{VO}_{2}\right)$ e produção de gás carbônico $\left.\left(\mathrm{VCO}_{2}\right)\right]$ durante o exercício na plataforma vibratória e na plataforma oscilatória, ambos utilizando o mesmo equipamento (Múltipla Fitness P500, Brasil). O equipamento possuiu três modalidades com potência determinada: a vibratória (300 Watts), a oscilatória (500 Watts) e a combinada (vibratória associada a oscilatória, 800 Watts)

As intervenções foram realizadas em dias alternados com um período de intervalo mínimo de três dias. Primeiramente os indivíduos foram submetidos a intervenção na plataforma vibratória e posteriormente a intervenção na plataforma oscilatória. Previamente a realização do exercício em ambas as modalidades foi conectado um analisador de gases (Total Metabolic Analysis System, TEEM 100, Aero Sport, Ann Arbor, Michigan). A intervenção teve um período de exercício de nove minutos, sendo realizada um minuto em cada posição ortostática: $120^{\circ}, 150^{\circ}$ e $180^{\circ}$ e de flexão de joelho, repetindo-se três vezes a sequência de exercícios, até totalizar nove minutos de exercício. Após finalizar o exercício manteve-se o equipamento de análise de gases conectado aos indivíduos por mais três minutos, os quais foram solicitados a permanecer na posição sentada neste período, para coleta dos dados referente a recuperação.

As variáveis hemodinâmicas, respiratórias e ventilatórias foram monitoradas antes (basal), durante o exercício (a cada minuto) e durante mais três minutos após o término do exercício (recuperação). A PAS e PAD foi mensurada com um esfignomanômetro (Bic, Brasil). A FC foi determinada usando o intervalo R-R a partir de 12 derivações do eletrocardiograma (Micromed, com software ErgoPc). A saturação periférica de oxigênio $\left(\mathrm{SpO}_{2}\right)$ foi monitorada com oxímetro de pulso (Onix 9500, Nonin, SIMS BCI Inc., Waukesha, WI, USA) e o esforço percebido pelo indivíduo foi mensurado pela escala analógica de Borg (Borg de membro inferior e Borg dispnéia), sendo esta uma mensuração do esforço percebido, por indicar com validade e confiança a monitorização da tolerância de um indivíduo ao exercício, também usada com frequência na realização de testes de esforço incremental, tendo relação com a FC e com a intensidade do exercício ${ }^{15}$.

As variáveis do teste foram calculadas como descrito previamente ${ }^{16}$. Em resumo, $\mathrm{VO}_{2}$ pico foi definido como o mais alto valor alcançado durante o teste por 20 segundos, e a potência circulatória de pico foi calculada como produto do $\mathrm{VO}_{2}$ pico e PAS de pico. A inclinação da ventilação $\left(\mathrm{V}_{\mathrm{E}}\right)$ e a produção de dióxido de carbono (VE/ $/ \mathrm{CO}_{2}$-inclinação) foi obtida através do modelo de regressão linear, usando os dados obtidos durante todo o teste e a amplitude relativa da oscilação na $V_{E}$ foi calculada a cada 20 segundos como a razão entre a amplitude e a média respectiva durante todo o teste. A eficiência da inclinação de consumo de oxigênio (OUES) foi calculada como a inclinação da linha de regressão linear entre $\mathrm{VO}_{2}$ e o logaritmo da $\mathrm{V}_{\mathrm{E}}{ }^{17}$. A cinética de recuperação do consumo de oxigênio foi avaliada como o tempo requerido para $50 \%$ de decréscimo a partir do $\mathrm{VO}_{2}$ pico $\left(\mathrm{T}_{1 / 2} \mathrm{VO}_{2}\right)$ e calculado usando o modelo matemático do quadrado mínimo, como descrito anteriormente ${ }^{16}$.

Para a análise dos dados foi utilizado o programa estatístico Statistical Package for Social Science - SPSS (versão 18.0, Chicago, IL, EUA). Na análise estatística todas as variáveis foram testadas quanto à normalidade pelo teste de Shapiro-Wilk. As variáveis quantitativas foram apresentadas por média \pm desvio padrão. Na comparação entre os valores obtidos ao longo do exercício na plataforma vibratória e oscilatória, utilizou-se o teste ANOVA medidas repetidas com teste post hoc de Bonferroni. Para comparação das médias das variáveis hemodinâmicas, respiratórias e ventilatórias entre a plataforma vibratória e oscilatória utilizou-se teste t pareado. Todos os testes foram aplicados com intervalo de confiança (IC) de 95\%. Considerou-se estatisticamente significativo os valores com $p<0,05$.

\section{RESULTADOS}

Um total de dez indivíduos saudáveis foram avaliados e realizaram ambos os protocolos de exercício na plataforma vibratória e oscilatória. A média de idade dos indivíduos foi de 23,0 $0 \pm 3,0$ anos, massa corporal média de $67,5 \pm 13 \mathrm{~kg} \mathrm{e}$

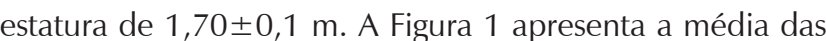
variáveis hemodinâmicas e respiratórias pré, durante e após o exercício na plataforma vibratória e oscilatória.

Como mostra a Figura 1, o treinamento na plataforma mantém uma PAS, PAD, $\mathrm{SpO}_{2}$ e Borg $\mathrm{P}$ similar entre as modalidades terapêuticas no decorrer do teste de exercício. A FC, por outro lado, foi maior na plataforma vibra- 
tória comparada a plataforma oscilatória, com diferença estatisticamente significativa nos tempos 1 e $3 \mathrm{~min}$. de exercício (Tabela 1).

A Tabela 1 apresenta as médias e desvios padrão destas variáveis e a comparação intra e intergrupos. Observa-se que a PAS e FC apresentaram alterações significativas intragrupos ao longo do teste de exercício. A PAS no $3^{\circ}$ e e $4^{\circ}$ min. de exercício foi significativamente superior a PAS do 3 o $\mathrm{min}$. de recuperação na plataforma vibratória, assim como, a FC do $1^{\circ}$ ao $8^{\circ} \min$. de exercício comparada a FC do $2^{\circ}$ e $3^{\circ}$ min. de recuperação e a $\mathrm{FC}$ do 3 o e 9 o min. de exercício comparada a FC de repouso. Na plataforma oscilatória, a PAS no $6^{\circ}$ min. de exercício foi significativamente superior a PAS do $3^{0}$ min. de recuperação, assim como, a PAS no minuto final de exercício comparada a PAS de repouso, a FC no 1 ,

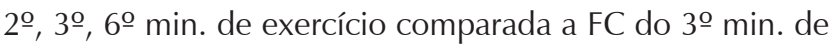
recuperação e a FC no 7 o min. de exercício comparado a FC do período de recuperação.

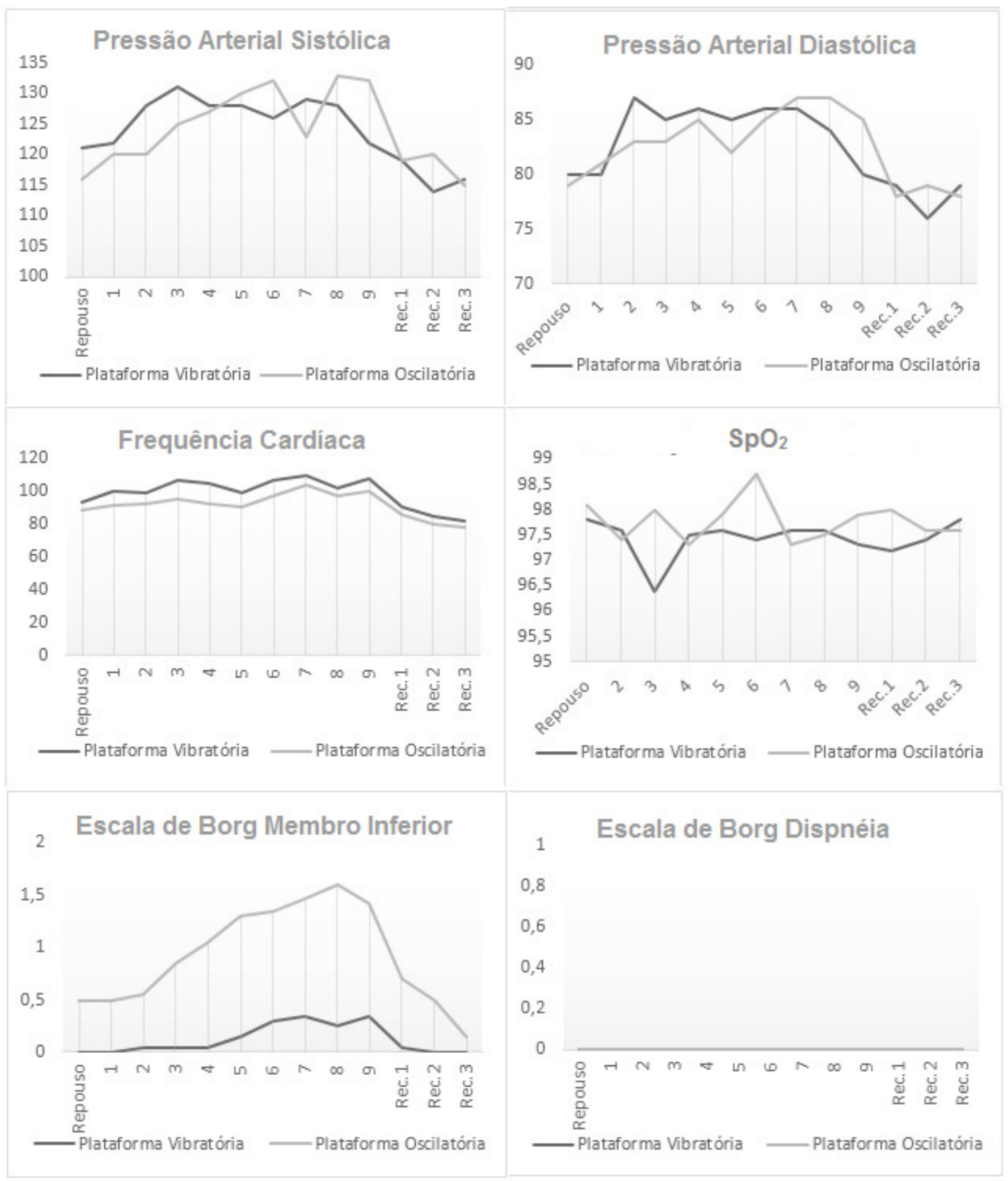

Figura 1. Média das variáveis hemodinâmicas e respiratórias pré, durante e após o exercício na plataforma vibratória e oscilatória. 
Tabela 1. Comparação das médias das variáveis hemodinâmicas e respiratórias durante o teste do exercício na plataforma vibratória e oscilatória.

\begin{tabular}{|c|c|c|c|c|c|c|c|c|c|c|c|c|c|c|c|}
\hline & \multicolumn{2}{|c|}{ PAS (mmHg) } & \multirow{2}{*}{$\underset{\text { inter }}{p}$} & \multicolumn{2}{|c|}{ PAD $(\mathrm{mmHg})$} & \multirow{2}{*}{$\underset{\text { inter }}{\mathbf{p}}$} & \multicolumn{2}{|c|}{ FC (bpm) } & \multirow{2}{*}{$\underset{\text { inter }}{\mathbf{p}}$} & \multicolumn{2}{|c|}{ SpO2 (\%) } & \multirow{2}{*}{$\underset{\text { inter }}{\mathbf{p}}$} & \multicolumn{2}{|c|}{ Borg MI } & \multirow{2}{*}{$\underset{\text { inter }}{\mathbf{p}}$} \\
\hline & Pvibrat. & Poscil. & & Pvibrat. & Poscil. & & Pvibrat. & Poscil. & & Pvibrat. & Poscil. & & Pvibrat. & Poscil. & \\
\hline Repouso & $119,0 \pm 11,0$ & $116,0 \pm 7,0$ & 0,468 & $79,0 \pm 9,9$ & $79,0 \pm 3,2$ & 1,0 & $93,5 \pm 18,1$ & 12,7 & 0,165 & $97,8 \pm 1,6$ & $98,1 \pm 1,1$ & 0,627 & $0,00 \pm 0,00$ & 0,50 & 0,177 \\
\hline 1 minuto & $121,0 \pm 11,0$ & $120,0 \pm 4,7$ & 0,780 & $80,0 \pm 4,7$ & $81,0 \pm 3,2$ & 0,678 & $100,6 \pm 18,1 \| \ddagger$ & $92,0 \pm 11,2 \ddagger$ & $0,049^{*}$ & $97,9 \pm 1,3$ & $97,7 \pm 1,5$ & 0,726 & $00 \pm 0,00$ & $0,50 \pm 1,08$ & 0,177 \\
\hline 2 minutos & $122,0 \pm 10,3$ & $120,0 \pm 4,7$ & 0,555 & $80,0 \pm 4,8$ & $83,0 \pm 6,7$ & 0,193 & $99,2 \pm 18,4 \| \ddagger$ & $92,7 \pm 14,6 \neq$ & 0,153 & $97,6 \pm 1,6$ & $97,4 \pm 2,7$ & 0,828 & $0,05 \pm 0,15$ & $0,55 \pm 1,06$ & 0,186 \\
\hline 3 minutos & $128,0 \pm 12,3 \neq$ & $125,0 \pm 5,3$ & 0,434 & $87,0 \pm 13,7$ & $83,0 \pm 6,7$ & 0,462 & $107,0 \pm 18,5 \# \| \neq$ & $95,3 \pm 9,8 \ddagger$ & $0,020^{*}$ & $96,4 \pm 3,1$ & $98,0 \pm 0,9$ & 0,153 & $0,05 \pm 0,15$ & $0,85 \pm 1,59$ & 0,157 \\
\hline 4 minutos & $131,0 \pm 11,0 \neq$ & $127,0 \pm 8,2$ & 0,269 & $85,0 \pm 8,5$ & $85,0 \pm 8,5$ & 1,0 & $105,4 \pm 15,3 \| \ddagger$ & $93,0 \pm 14,7$ & 0,073 & $97,5 \pm 1,6$ & $97,3 \pm 3,0$ & 0,859 & $0,05 \pm 0,15$ & $1,05 \pm 2,52$ & 0,246 \\
\hline 5 minutos & $128,0 \pm 9,2$ & $130,0 \pm 12,5$ & 0,642 & $86,0 \pm 7,0$ & $82,0 \pm 7,8$ & 0,168 & $99,5 \pm 18,0 \| \neq$ & $90,6 \pm 14,3$ & 0,086 & $97,6 \pm 1,3$ & $97,9 \pm 2,2$ & 0,745 & $0,15 \pm 0,33$ & $1,30 \pm 3,12$ & 0,275 \\
\hline 6 minutos & $128,0 \pm 15,5$ & $132,0 \pm 11,3 \ddagger$ & 0,462 & $85,0 \pm 9,7$ & $85,0 \pm 7,1$ & 1,0 & $107,5 \pm 20,0 \| \ddagger$ & $97,1 \pm 12,3 \neq$ & 0,123 & $97,4 \pm 1,3$ & $98,7 \pm 2,3$ & 0,128 & $0,30 \pm 0,48$ & $1,35 \pm 2,23$ & 0,164 \\
\hline 7 minutos & $126,0 \pm 15,8$ & $123,0 \pm 30,6$ & 0,806 & $86,0 \pm 8,4$ & $87,0 \pm 9,5$ & 0,798 & $110,1 \pm 18,1 \| \ddagger$ & $103,8 \pm 16,2 \mid \| \ddagger$ & 0,175 & $97,6 \pm 1,8$ & $97,3 \pm 2,3$ & 0,755 & $0,35 \pm 0,41$ & $1,47 \pm 2,84$ & 0,258 \\
\hline 8 minutos & $129,0 \pm 14,5$ & $133,0 \pm 11,6$ & 0,534 & $86,0 \pm 9,7$ & $87,0 \pm 10,6$ & 0,832 & $102,6 \pm 18,7 \| \ddagger$ & $97,5 \pm 16,7$ & 0,207 & $97,6 \pm 1,7$ & $97,5 \pm 3,1$ & 0,939 & $0,25 \pm 0,42$ & $1,60 \pm 3,13$ & 0,205 \\
\hline 9 minutos & $128,0 \pm 16,9$ & $132,0 \pm 9,2 \#$ & 0,555 & $84,0 \pm 8,4$ & $85,0 \pm 5,3$ & 0,758 & $107,6 \pm 17,9 \#$ & $100,7 \pm 11,4$ & 0,136 & $97,3 \pm 1,1$ & $97,9 \pm 1,2$ & 0,279 & $0,35 \pm 0,47$ & $1,42 \pm 2,35$ & 0,175 \\
\hline Rec. $1 \mathrm{~min}$. & $122,0 \pm 14,8$ & $119,0 \pm 11,9$ & 0,627 & $80,0 \pm 9,4$ & $78,0 \pm 9,2$ & 0,619 & $91,1 \pm 12,5$ & $85,8 \pm 13,1$ & 0,099 & $97,2 \pm 1,4$ & $98,0 \pm 0,6$ & 0,087 & $0,05 \pm 0,15$ & $0,70 \pm 1,33$ & 0,169 \\
\hline Rec. 2 min. & $119,0 \pm 12,9$ & $120,0 \pm 14,1$ & 0,879 & $79,0 \pm 7,4$ & $79,0 \pm 7,4$ & 1,0 & $84,9 \pm 14,6$ & $80,0 \pm 11,2$ & 0,245 & $97,4 \pm 1,0$ & $97,6 \pm 1,0$ & 0,555 & $0,00 \pm 0,00$ & $0,50 \pm 1,08$ & 0,177 \\
\hline Rec. $3 \mathrm{~min}$. & $114,0 \pm 10,7$ & $115,0 \pm 9,7$ & 0,853 & $76,0 \pm 5,2$ & $78,0 \pm 6,3$ & 0,555 & $82,6 \pm 13,6$ & $78,7 \pm 12,5$ & 0,200 & $97,9 \pm 1,2$ & $97,6 \pm 0,7$ & 0,591 & $0,00 \pm 0,00$ & $0,15 \pm 0,33$ & 0,193 \\
\hline$p$ intragru & 0,005 & 0,002 & & 0,093 & 0,104 & & 0,001 & 0,001 & & 0,395 & 0,651 & & 0,092 & 0,182 & \\
\hline
\end{tabular}

*p significativo intergrupos. \#p versus repouso; |p versus recuperação 1 minutos; ||p versus recuperação 2 minutos; łp versus recuperação 3 minutos. Dados apresentados em média e desvio padrão. PAS: pressão arterial sistólica; mmHg: milímetros de mercúrio; PAD: pressão arterial diastólica; FC: frequência cardíaca; bpm: batimentos por minuto; $\mathrm{SpO}_{2}$ : saturação periférica de oxigênio; \%: percentual; Borg MI: nível subjetivo de fadiga de membros inferiores; minutos: min.

Com relação as variáveis ventilatórias, o exercício na plataforma oscilatória mantém um $\mathrm{VO}_{2}$ e $\mathrm{VCO}_{2}$ superior comparado ao exercício na plataforma vibratória (Figura 2), com diferença estatisticamente significativa nos tempos 4,
5 e 7 min. de exercício para o $\mathrm{VO}_{2}$ e nos tempos 4, 5, 7, 8 e 9 para o $\mathrm{VCO}_{2}$. A Tabela 2 apresenta as médias e desvios padrão destas variáveis e a comparação intra e intergrupos. Observa-se que o $\mathrm{VO}_{2}$ e o $\mathrm{VCO}_{2}$ apresentaram alterações

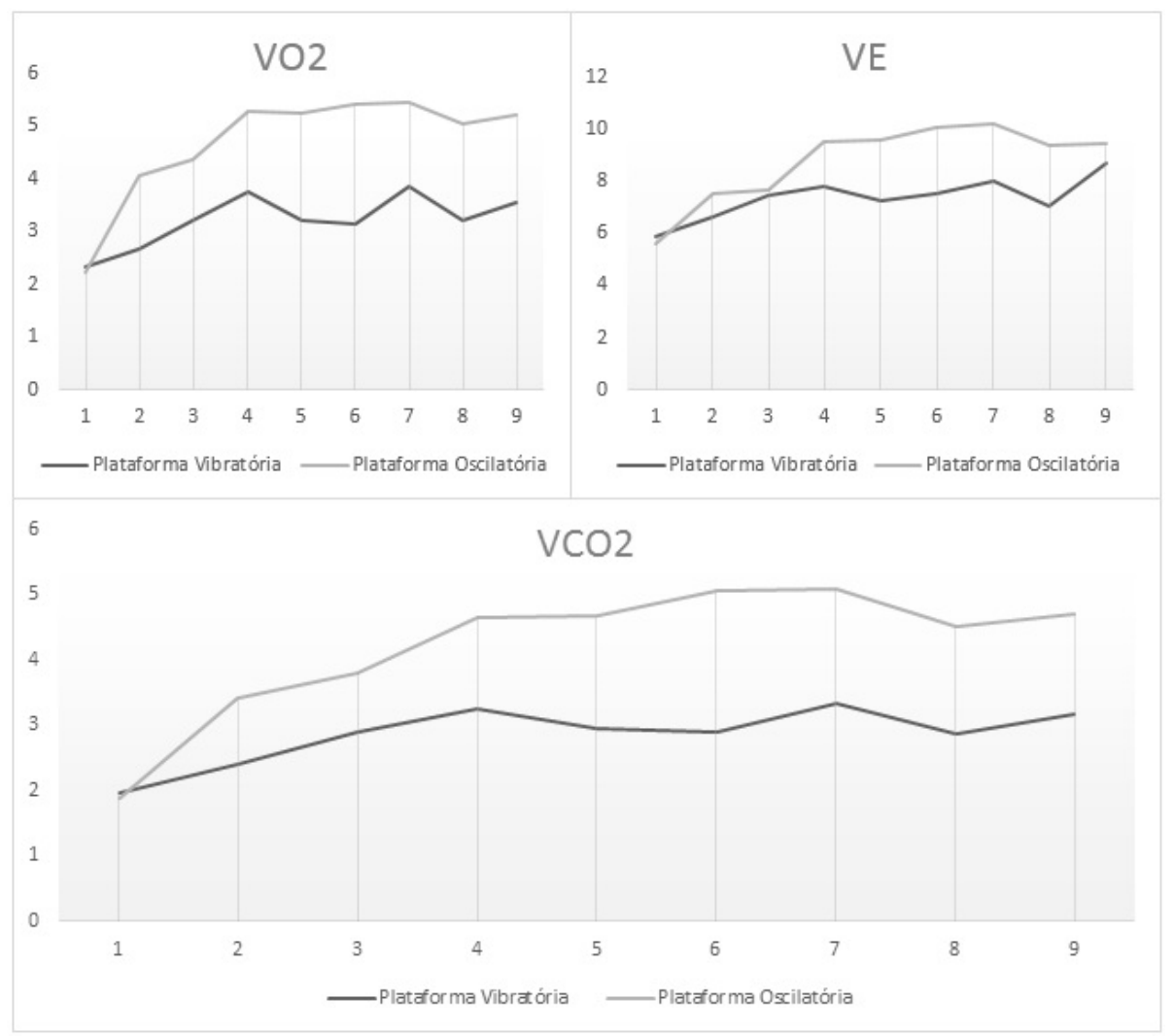

Figura 2. Média das variáveis ventilatórias durante o teste de exercício na plataforma vibratória e oscilatória. 
significativas intragrupos ao longo do teste de exercício. O $\mathrm{VO}_{2}$ no 8 o min. de exercício foi significativamente superior ao $\mathrm{VO}_{2}$ no final do exercício na plataforma vibratória. $\mathrm{Na}$ plataforma oscilatória, o $\mathrm{VO}_{2}$ no $3 \stackrel{\circ}{\circ}, 4 \stackrel{\circ}{\circ}, 6$ o e $7 \stackrel{0}{\circ}$ min. de exercício foi significativamente superior ao $\mathrm{VO}_{2}$ de repouso, assim como, o $\mathrm{VCO}_{2}$ no 8 o min. de exercício comparada ao $\mathrm{VCO}_{2}$ de repouso.
A Tabela 3 mostra os resultados obtidos através do analisador de gases coletado durante o exercício na plataforma vibratória e oscilatória. O exercício na plataforma oscilatória evidencia valores significativamente superiores de $\mathrm{VO}_{2}$ pico, $\mathrm{VCO}_{2}$ pico e potência circulatória pico quando comparado ao exercício na plataforma vibratória.

Tabela 2. Comparação das médias das variáveis ventilatórias pré, durante e ao término do teste do exercício e entre as modalidades da plataforma: vibratória e oscilatória.

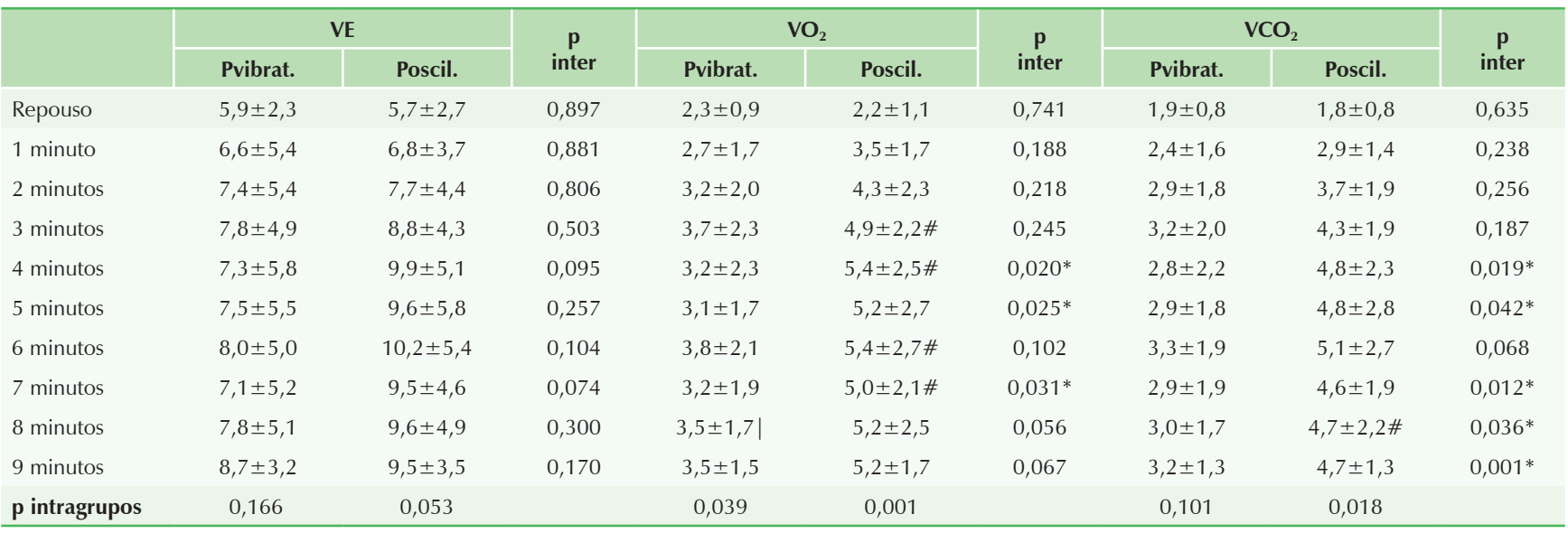

*p significativo intergrupos; \#p significativo versus repouso; | p significativo versus final do exercício. Dados apresentados em média e desvio padrão. VE: ventilação; $\mathrm{O}_{2}$ : consumo de oxigênio; $\mathrm{VCO}_{2}$ : produção de dióxido de carbono.

Tabela 3. Resultados obtidos no teste ergoespirométrico na plataforma vibratória e oscilatória.

\begin{tabular}{|c|c|c|c|}
\hline & $\begin{array}{l}\text { Plataforma } \\
\text { Vibratória }\end{array}$ & $\begin{array}{l}\text { Plataforma } \\
\text { Oscilatória }\end{array}$ & p \\
\hline \multicolumn{4}{|l|}{ Pico do exercício } \\
\hline Frequência cardíaca pico (bpm) & $114,1 \pm 17,9$ & $107,4 \pm 14,8$ & 0,079 \\
\hline Pressão sistólica pico (mmHg) & $137,0 \pm 13,4$ & $136,0 \pm 12,6$ & 0,832 \\
\hline $\mathrm{VO}_{2}$ pico $\left(\mathrm{mL} \cdot \mathrm{kg}^{-1} \cdot \mathrm{min}^{-1}\right)$ & $5,2 \pm 2,1$ & $7,7 \pm 2,7$ & $0,012^{*}$ \\
\hline $\mathrm{VCO}_{2}$ pico $\left(\mathrm{L} \cdot \mathrm{min}^{-1}\right)$ & $4,5 \pm 2,1$ & $6,9 \pm 3,2$ & $0,035^{*}$ \\
\hline$V_{E}$ pico $\left(L \cdot \mathrm{min}^{-1}\right)$ & $10,8 \pm 5,8$ & $14,1 \pm 6,8$ & 0,068 \\
\hline R pico & $1,00 \pm 0,13$ & $0,99 \pm 0,15$ & 0,927 \\
\hline Potência circulatória pico, $\mathrm{mmHg} \cdot \mathrm{mL} \mathrm{O}{ }_{2} \cdot \mathrm{kg}^{-1} \cdot \mathrm{min}^{-1}$ & $736,1 \pm 338,9$ & $1066,0 \pm 466,2$ & $0,029^{*}$ \\
\hline \multicolumn{4}{|l|}{ Resposta Ventilatória } \\
\hline $\mathrm{VE} / \mathrm{VCO}_{2}$ - inclinação & $2202,6 \pm 881,3$ & $1773,3 \pm 442,8$ & 0,179 \\
\hline OUES, $\mathrm{mL} \cdot \mathrm{min}^{-1} \mathrm{O}_{2} /$ L. $\mathrm{min}^{-1} \mathrm{VE}$ & $0,006 \pm 0,003$ & $0,01 \pm 0,004$ & 0,051 \\
\hline Oscilação na $V_{E}$ & $27,8 \pm 23,2$ & $32,2 \pm 19,5$ & 0,596 \\
\hline \multicolumn{4}{|l|}{ Recuperação das trocas gasosas } \\
\hline $\mathrm{T}_{1 / 2} \mathrm{VO}_{2}$, seg & $68,0 \pm 16,9$ & $58,0 \pm 11,4$ & 0,177 \\
\hline
\end{tabular}

Dados apresentados em média e desvio padrão. Teste t pareado. $\mathrm{VO}_{2}$ pico: consumo de oxigênio de pico; $\mathrm{VCO}_{2}$ pico: produção de dióxido de carbono de pico; VE pico: ventilação de pico; R pico: razão de troca respiratória de pico; $V E / V \mathrm{VO}_{2}$ - inclinação: inclinação da linha de regressão das mudanças na $\mathrm{VE} \mathrm{e} \mathrm{VCO}_{2}$ durante o teste de treinamento; OUES: eficiência da inclinação de consumo de oxigênio; $\mathrm{T}_{1 / 2}=$ tempo requerido para queda $50 \%$ do $\mathrm{VO}_{2}$ a partir do pico. 


\section{DISCUSSÃO}

Esta pesquisa buscou avaliar os efeitos hemodinâmicos, respiratórios e ventilatórios durante o exercício na plataforma nas modalidades vibratória e oscilatória e comparar os efeitos entre as modalidades terapêuticas em indivíduos saudáveis, a fim de conhecer seus efeitos para a prescrição adequada e segura desta forma de exercício. É uma modalidade de exercício atualmente usada com grande frequência em academias e centros de reabilitação física para pacientes com alguma limitação física. Como estas modalidades de exercício são relativamente novas na prática clínica diária, percebe-se uma falta de informações sobre seus efeitos hemodinâmicos e ventilatórios.

No presente estudo verificou-se alterações fisiológicas durante o exercício nas variáveis hemodinâmicas (PAS e FC) e ventilatórias $\left(\mathrm{VO}_{2}\right.$ e $\left.\mathrm{VCO}_{2}\right)$ nas plataformas vibratória e oscilatória. Ao comparar o exercício entre as modalidades da plataforma, observou-se que a oscilatória tende a provocar um maior aumento no $\mathrm{VO}_{2}, \mathrm{VCO}_{2}$ e potência circulatória, comparada a modalidade vibratória. As alterações ocorridas são consideradas fisiológicas na vigência da realização de um exercício físico. Poucos estudos mostram os efeitos hemodinâmicos e metabólicos do exercício na plataforma a longo prazo ${ }^{18}$, e não existe relato até o momento, dentro da investigação realizada para este estudo, dos efeitos agudos desta modalidade de exercício. Por esta razão, este estudo buscou verificar os efeitos agudos do exercício durante as duas modalidades na plataforma (vibratória e oscilatória).

Os efeitos fisiológicos agudos durante o exercício nas variáveis hemodinâmicas, como pressão arterial sistêmica e frequência cardíaca, tendem a alterar. Em exercícios mais dinâmicos, envolvendo grandes grupos musculares, a pressão arterial e a FC aumentam em proporção direta ao aumento da intensidade do esforço. A PAS pode exceder $200 \mathrm{mmHg}$ no pico da sístole e no momento da exaustão ${ }^{19}$. No presente estudo, o treinamento na plataforma foi realizado em uma intensidade constante, mantendo a mesma frequência vibratória ou oscilatória, e com uma duração de nove minutos. Por esse motivo, a PAS atingiu o máximo de 131 e $133 \mathrm{mmHg}$ nas modalidades vibratória e oscilatória, respectivamente, havendo a recuperação aos valores de repouso após o término do exercício. Portanto, o treinamento provocou um aumento dentro de uma faixa segura. Ressaltase que foi um exercício de curta duração, não levando a exaustão do indivíduo o que pode ser confirmado pela baixa pontuação na percepção do esforço na escala de Borg e a baixa variação nas variáveis analisadas.

O aumento superior das variáveis ventilatórias ocorridas durante o exercício, na plataforma oscilatória quando comparada a plataforma vibratória, pode ser devido a maior frequência do equipamento, sendo 500 e 300 watts, respectivamente, gerando maior gasto metabólico o que foi demonstrado pelo maior consumo de oxigênio nesta modalidade da plataforma. $\mathrm{O} \mathrm{VO}_{2}$ pico é a maior taxa de consumo de oxigênio possível, e é obtido por meio de um teste incremental e considera-se 20 a $39 \mathrm{ml}^{\mathrm{kg}}{ }^{-1} \cdot \mathrm{min}^{-1}$ dentro dos parâmetros esperados para a população jovens saudável estudada ${ }^{20}$. A análise do exercício realizado na plataforma nas modalidades vibratória e oscilatória, não é considerada um teste incremental, mas um exercício de carga constante e baixa carga, pois o $\mathrm{VO}_{2}$ pico obtido foi em torno de $1 \mathrm{MET}$ para o exercício na plataforma vibratória e de 1,5 MET na plataforma oscilatória. Estes dados refletem que a plataforma é um ergômetro com baixa exigência aeróbia no protocolo que foi executado (nove minutos na posição em pé, alternando o posicionamento dos joelhos). Por isto, sugerese a realização de estudos, nesta temática, em pacientes com doenças crônicas não transmissíveis como Doença Pulmonar Obstrutiva Crônica, Doença de Parkinson, Acidente Vascular Encefálico, dentre outras a fim de contribuir com a discussão na literatura sobre este tipo de terapêutica e seus efeitos nas variáveis hemodinâmicas, respiratórias e ventilatórias.

$\mathrm{Na}$ análise das demais variáveis obtidas com o analisador de gases verificou-se que o $\mathrm{VO}_{2}$ pico foi maior na plataforma oscilatória, assim como a potência circulatória pico, porém esta última aumentou em decorrência do maior $\mathrm{VO}_{2}$ pico obtido no teste, visto que estas variáveis apresentaram uma forte correlação de Pearson ( $r=0,988 ; p<0,001)$, e a PASpico manteve-se praticamente a mesma nas duas modalidades da plataforma. É importante ressaltar que, no presente estudo, foi realizado uma análise das variáveis do analisador de gases incluindo eficiência da inclinação de consumo de oxigênio, eficiência ventilatória e oscilação da ventilação, bem como a cinética de recuperação do consumo de oxigênio durante o treinamento na plataforma vibratória e oscilatória, um exercício constante. A maioria dos estudos ${ }^{17,21,22}$ abordam estas variáveis durante o teste de exercício cardiopulmonar incremental, que é um teste de exercício máximo.

Os valores de FC e PAS em repouso foram inferiores, embora não significativo estatisticamente, no exercício realizado na plataforma oscilatória quando comparado a vibratória. Além disso, os valores de FC no 1 o e 3 o min. de exercício foram significativamente inferiores na plataforma oscilatória, comparada a vibratória. Isto pode ser atribuído a ansiedade provocada pelo exercício até então desconhecido pelos participantes do estudo, pois estes foram submetidos primeiramente ao exercício na plataforma vibratória e no segundo momento na oscilatória, no qual já estavam familiarizados com o equipamento e com o teste. Este 
aspecto poderá ser considerado uma das limitações do estudo, e poderia ser evitado se houvesse a randomização dos indivíduos para a realização das modalidades na plataforma.

Dentre as limitações deste estudo podemos atribuir o fato que os indivíduos não haviam realizado este tipo de exercício anteriormente a pesquisa e não foi realizado treinamento prévio antes da execução do protocolo de pesquisa o que poderia alterar parâmetros como a FC pela ansiedade dos participantes.

Os resultados mostram que o uso deste equipamento, independente da modalidade, durante o processo de reabilitação, não desencadeia alterações significativas a curto prazo, além das esperadas durante a realização de um exercício, nas respostas hemodinâmicas, respiratórias e ventilatórias em indivíduos jovens e saudáveis. A modalidade na plataforma oscilatória, ao ser comparada com a vibratória, proporciona um aumento superior das respostas ventilatórias. Sugere-se que mais estudos com outras populações sejam realizados a fim de fortalecer a discussão desta modalidade terapêutica atualmente realizada em academias e Centros de Reabilitação Física.

\section{REFERÊNCIAS}

1. Pardo EM, Páez LC, Ramón PEA, Gómez AB, Soler CN. Efectos agudos de las vibraciones mecánicas sobre el salto vertical. Ed Fís y Depor. 2007;87:81-5.

2. Kelley GA, Kelley KS. Efficacy of aerobic exercise on coronary heart disease risk factors. Prev Cardiol. 2008;11(2):71-5. http://dx.doi. org/10.1111/j.1751-7141.2008.08037.x

3. Hills AP, Street SJ, Byrne NM. Physical Activity and Health: "What is Old is New Again". Adv Food Nutr Res. 2015;75:77-95. http:// dx.doi.org/10.1016/bs.afnr.2015.06.001

4. Buzzachera CF, Elsangedy HM, Hallage T, Silva SG. Parâmetros fisiológicos e perceptivos durante caminhada de intensidade preferida por mulheres adultas, previamente sedentárias. Rev Bras Cineantr Desempenho Hum. 2007;9:170-6.

5. Rittweger J, Ehrig J, Just K, Mutschelknauss M, Kirsch KA, Felsenberg D. Oxygen uptake in whole-body vibration exercise: influence of vibration frequency, amplitude, and external load. Int J Sports Med. 2002;23(6):428-32. http://dx.doi.org/10.1055/s-2002-33739

6. Verschueren SM, Roelants M, Delecluse C, Swinnen S, Vanderschueren D, Boonen S. Effect of 6 month whole body vibration training on hip density, muscles strength, and postural control in postmenopausal women: a randomized controlled pilot study. J Bone Miner Res. 2004;19(3):352-9. http://dx.doi. org/10.1359/JBMR.0301245

7. Baum K, Votteler T, Schiab J. Efficiency of vibration exercise for glycemic control in type 2 diabetes patients. Int J Med Sci. 2007;4(3):159-63. http://dx.doi.org/10.7150/ijms.4.159

8. Bogaerts A, Delecluse C, Claessens AL, Coudyzer W, Boonen S, Verschueren SM. Impact of Whole-Body Vibration Training Versus
Fitness Training on Muscle Strength and Muscle Mass in Older Men: A 1-Year Randomized Controlled Trial. J Gerontol A Biol Sci Med Sci. 2007;62(6): 630-5. http://dx.doi.org/10.1093/gerona/62.6.630

9. Anderson K; Behm DG. The impact of instability resistance training on balance and stability. Sports Med. 2005;35(1):45-53. http:// dx.doi.org/10.2165/00007256-200535010-00004

10. Borghuis J, Hof AL, Lemmink KA. The importance of sensory-motor control in providing core stability implications for measurement and training. Sports Med. 2008;38(11):893-916. http://dx.doi. org/10.2165/00007256-200838110-00002

11. Hill KD, Logiudice D, Lautenschlager NT, Said CM, Dodd KJ, Suttanon P. Effectiveness of balance training exercise in people with mild to moderate severity Alzheimer's disease: protocol for a randomized trial. BMC Geriatr. 2009;9:29. http://dx.doi.org/10.1186/14712318-9-29

12. Rasool J, George K. The impact of single-leg dynamic balance training on dynamic stability. Phys Ther Sport. 2007;8:177-84. http://dx.doi.org/10.1016/j.ptsp.2007.06.001

13. Laudner KG, Koschnitzky MM. Ankle muscle activation when using the Both Sides Utilized (BOSU) balance trainer. J Strength Cond Res. 2010;24(1):218-22. http://dx.doi.org/10.1519/ JSC.0b013e3181c490d4

14. Yaggie JA, Campbell BM. Effects of balance training on selected skills. J Strength Cond Res. 2006;20(2):422-8.

15. American College of Sports Medicine. Diretrizes do ACSM para os testes de esforço e sua prescrição. Rio de Janeiro: Guanabara Koogan; 2003.

16. Dall'Ago P, Chiappa GR, Guths H, Stein R, Ribeiro JP. Inspiratory muscle training in patients with heart failure and inspiratory muscle weakness. A randomized trial. J Am Coll Cardiol. 2006;47(4): 757-63. http://dx.doi.org/10.1016/j.jacc.2005.09.052

17. Gademan MG, Swenne CA, Verwey HF, van de Vooren $\mathrm{H}$, Haest JC, van Exel HJ, Lucas CM, Cleuren GV, Schalij MJ, van der Wall EE. Exercise training increases oxygen uptake efficiency slope in chronic heart failure. Eur J Cardiovasc Prev Rehabil. 2008;15(2):140-4. http://dx.doi.org/10.1097/HJR.0b013e3282ef19986

18. Pessoa MF. Efeitos cardiopulmonares do treinamento em plataforma vibratória em idosos saudáveis [dissertação]. Recife: Universidade Federal de Pernambuco; 2012.

19. Fisiologia do exercício. Brasília: Fundação Vale; 2013.

20. Neder JA, Nery LE, Castelo A, Andreoni S, Lerario MC, Sachs A, Silva AC, Whipp BJ. Prediction of metabolic and cardiopulmonary responses to maximum cycle ergometry: a randomized study. Eur Respir J. 1999;14(6):1304-13. http://dx.doi.org/10.1183/09031936. 99.14613049

21. Winkelmann ER, Chiappa GR, Lima CO, Viecili PR, Stein R, Ribeiro $J P$. Addition of inspiratory muscle training to aerobic training improves cardiorespiratory responses to exercise in patients with heart failure and inspiratory muscle weakness. Am Heart J. 2009;158(5):768. e1-7. http://dx.doi.org/10.1016/j.ahj.2009.09.005

22. Zurek M, Binder RK, Saner H, Schmid J-P. Exercise training reduces oscillatory ventilation during exercise. Eur J Cardiovasc Prevent Rehabil 2008;15(Suppl 1):S74. 